Fathers who use domestic violence: Organisational capacity building and practice development

Cathy Humphreys, Professor of Social Work, University of Melbourne

Dr Lucy Healey, Senior Research Fellow, Department of Social Work, University of Melbourne

Dr Susan Heward-Belle, School of Education and Social Work, University of Sydney

Correspondence:

Cathy Humphreys

Professor of Social Work

University of Melbourne

Parkville, Victoria,

Australia. 3010

Cathy.humphreys@unimelb.edu.au

Ph. 61-3-83442729

This is the author manuscript accepted for publication and has undergone full peer review but has not been through the copyediting, typesetting, pagination and proofreading process, which may lead to differences between this version and the Version of Record. Please cite this article as doi: $10.1111 / \mathrm{cfs} .12708$

This article is protected by copyright. All rights reserved. 


\section{Fathers who use domestic violence: Organisational capacity building and practice development}

\section{Abstract}

It is well established in the literature that fathers who use violence harm women and children. Statutory child protection workers and other human service professionals now face strong injunctions to work with these men. However, insufficient attention has been given to considering the processes for intervening with fathers who use violence, and the organisational context to support workers in refocusing their practice. Workers lack clear guidance and organisations lack specific understandings of how to support workers in this complex area of practice. Australian research used co-design with professionals in Communities of Practice, supported by the Safe\& Together Institute to explore the organisational context required to work with fathers who use violence. This paper presents findings of qualitative data gathered from surveys, focus groups and ethnographic notes of 30 Community of Practice sessions. Working together, practitioners and researchers identified four essential elements to support organisational development and improve practice: leadership by senior managers; recognition that domestic violence work needs to strengthen collaborative working; extensive training, supervision and coaching; and enhanced worker safety. Worker perspectives are critical in developing practice principles and identifying organisational mechanisms that support practitioners to work safely and effectively with fathers who use violence.

\section{Introduction}

Recognition that men who use violence in their interpersonal relationships are frequently fathers who parent or have ongoing contact with their children post separation has received little attention. Service responses tend to focus on intervening with women as victims/survivors and as mothers (Witt \& Diaz, 2019), rather than on the parenting capacity of fathers who use domestic violence (DV) (Anonymous, 2018; Nygren et al, 2019). The skills, knowledge and organisational support that 
statutory social workers and other human service workers require to investigate, assess and engage with fathers who use violence is under-developed. There are now strong injunctions to keep the perpetrator of violence in view and for workers to focus attention on identifying and redressing the harm to children and their mothers created by men who use violence (Anonymous, 2019). However, few workers are trained to undertake work with men who use violence particularly in relation to their parenting. Furthermore, the organisational support needed to address this practice is also underdeveloped.

Clearly terminology issues are important. We have primarily used the terminology of 'fathers who use violence' to highlight the fact that domestic violence (DV) is not only an adult problem of 'men who use violence', but that when children are involved their interpersonal violence shapes their role as fathers. Gendered terminology is used to reflect the dominant pattern of DV as violence by men towards women and their children (Cox, 2016)

This paper reports on Australian research, Invisible practices: Working with fathers who use violence, which explored the elements contributing to practice development in working with fathers who use violence, including the organisational context required to support practice change. Communities of Practice (Blackmore, 2010) with practitioners were developed as the central element in an action research process to co-design the organisational responses required for practice change (Evans \& Terrey, 2016).

\section{Literature review}

The literature about fathers who use violence and organisational change foreground this research. The interconnection between these areas recognises that practitioners work within organisations and that individual practice cannot be isolated from its context (Healy, 2017). The link between understanding the impact of fathers' violence on children has led to demands for statutory child 
protection social workers, family service workers and specialist DV workers to 'pivot to the perpetrator' (Mandel, 2014) and re-orientate practice which has tended to ignore fathers and their impact on children (Nygren et al, 2019; Scourfield et al, 2015). However, statutory child protection practice has generally been orientated to the child's mother and her ability to protect her children from violence, rather than using the leverage of statutory involvement to assess and work with fathers who use violence (Anonymous, 2018a).

The evidence of destructive consequences for children's exposure to DV is compelling and well established (McTavish et al, 2016). Negative health impacts (Riviara et al, 2007), undermining of children's emotional and psychological well-being (Holt et al, 2008), and problems associated with destructive behaviours have been consistently identified. Evidence highlights that both adult and child victims exist where DV occurs. While there is widespread recognition that DV is predominantly a gendered issue of men's violence towards women (Cox, 2016), less attention is given to children as victims of the destructive parenting behaviours of domestically violent fathers.

Anonymous (2015) argued that the parenting knowledge and skills of domestically violent fathers is under-developed. The controlling behaviours used against partners may also be deployed against children (Holt, 2015). The physical violence used towards their partners is reflected in the greater use of smacking, and heightened rates of physical abuse of their children (Anonymous, 2019). Fathers' accounts of their use of violence range from using violence instrumentally to using violence uncontrollably, with equally destructive impacts on children (Anonymous, 2015). DV also undermines the child's mother through physical and sexual assault, psychological tactics of coercion, denigration of her mothering (Buchanan, 2018) and the inability of fathers who use violence to coparent (Thompson-Walsh et al, 2018).

However, the practice in working with fathers who use violence by statutory child protection social workers and other human service professionals requires greater attention. Most of the practice development in working with fathers who use violence has been based within single sex, 
group work programs such as the Jacana project or Caring Dads (Coy et al, 2011; McConnell \& Taylor, 2016). A scoping review which explored the practice of workers from different professions who see men who use violence outside group work programs revealed few evaluated initiatives but emergent practice guidelines and pilot programs (Anonymous, 2017b).

The knowledge base about the negative impact of fathers who use violence on children has outstripped practice and organisational development particularly for child protection workers who are regularly requested to investigate, assess and intervene in these families, but without the necessary skills and organisational support to do this work (Anonymous, 2019). Practice change is required. However, there are many factors involved for change to become embedded. Organisational leadership and the context within which change occurs are critical (Gilley et al, 2009). Innovations require an authorising environment which allows for systematic changes in the structure and delivery of services. (May, 2013). The complexity of organisational change is not to be underestimated (Ison, 2017).

In the process of organisational change, co-design may have a role to play. Co-design is both a methodology for research and professional reflection (Evans \& Terrey, 2016). The first stage of codesign involves discovery, insight and the development of a shared language based on an agreed understanding of issues from participants. The development of prototypes or consensus ways of working are then created with a view to evaluation as ideas are taken to scale (Evans \& Terrey, 2016).

Current work on co-design has largely focused on end users of services, particularly in the health area (Gustavsson \& Andersson, 2017). However, a case can be made for involving professionals in redesigning their work environment to create more effective responses (Howard \& Somerville, 2014). When workers are asked to embark upon practice that increases the risks to their emotional and physical well-being through working with fathers who use violence, it is arguably essential for them to be key players in designing the ways in which this might safely occur. Through 
'practice-led knowledge building', their experience and knowledge of legislation, policies and procedures which configures their practice can contribute to new ways of working (Wagenaar \& Cook, 2011). The lacunae in practice-led knowledge development motivated the research team to explore the question: What do practitioners require from organisations to support them in working with fathers who use violence?

\section{Research Design}

An action research approach provided the framework for a complex research design which moved through iterative processes simultaneously contributing to practice change and evidence gathering (Ison, 2008). The resources and expertise of the Safe\&Together Institute and the development of Communities of Practice (CoPs) and Project Advisory Groups were integral to the research design. The research methods included: ethnographic note-taking during Community of Practice(CoP) and Program Advisory Group meetings; Time 1 and Time 2 online questionnaires; and focus groups.

\section{The Safe\&Together ${ }^{\text {TM} M o d e l ~}$}

To contribute to the skills and knowledge of practitioners, a leading practitioner/consultant in DV and child protection, David Mandel, and his colleagues from the Safe\&Together Institute partnered with the research team. It was envisaged that Safe\&Together expertise, resources, training and coaching would build the capacity of local practitioners. The Safe\&Together Model provides a framework and resources to guide DV practice where there are children involved. The framework is simple, but the practice complex (Mandel, 2009). While the principles underpinning the model are well established, the development of practice, the subsequent increase in resources and evidence to support practice are original contributions that are shifting organizational processes and professional practice. It is here that co-design of new guidance in particular areas has a role to play, and it was agreed that a focus on fathers who use violence and engagement in relation to their parenting practices was an area for development. 
The basic principles are to: pivot to the perpetrator; build an alliance with the non-offending parent (usually mother); focus on the child/ren; and explore the complexity of DV and its interaction with mental health, substance misuse, and other intersections such as culture, poverty and disability. A focus of the work is strengthening documentation of harm to the child and family functioning created through the perpetrator's pattern of coercive control and clearly documenting the non-offending parent's protective actions (Safe\&Together Institute, 2018).

The framework emphasises that organisational culture is central to framing the context for changes to practice for individual practitioners (Anonymous, 2018). Individual training, coaching and supervision cannot be embedded without a supportive environment in which to engage in new practices. Hence, a central focus of the research (and this article) lies in the exploration of organisational context as the avenue for capacity building practitioners.

\section{Establishment of Communities of Practice}

The establishment of CoPs provided the engine for co-design and a foundation for the research. Researchers from four Australian states with expertise in DV and child protection research and longstanding relationships with government and NGOs designed the project with the following components (see Figure 1):

- A Program Advisory Group for each state site comprised of senior managers from government departments, NGOs and peak bodies. As significant project stakeholders, Program Advisory Groups provided the authorising environments for practitioners to participate in a CoP and offered strategic advice about how to drive changes recommended by practitioners.

- The establishment of CoPs in each state with at least two senior practitioners from each participating organisation. 34 child protection workers; 14 specialist DV workers; and 14 workers from family services and 5 other workers from health and justice.

This article is protected by copyright. All rights reserved. 
- Video-conference consultations from Safe\&Together experts to CoP practitioners.

Figure 1: Type and number of participants in Invisible Practices project

[place here]

Funding was provided by the Australian National Research Organisation for Women's Safety (ANROWS) and participating state government departments. Ethical clearance was provided through the host university and expectations for CoP members were outlined in the Plain Language Statement (ID 1749310).

The CoPs consisted of six state-based, two-hour, monthly workshops in four participating states. CoPs are generally established to share knowledge and acquire skills by working collectively and regularly on a shared problem or challenge (Blackmore, 2010). In keeping with a co-design framework, CoPs provide an avenue for building consensus on a shared framework and language in responding to an identified issue, namely the development of principles for working with domestically violent fathers and establishing the organisational context to enable this work.

One state-based researcher documented the practice and policy issues raised by practitioners and created ethnographic notes to capture the development of a shared language and approach at each CoP. Ground rules established CoPs as safe and respectful workspaces, devoid of judgement on individual practice, focused on insights into the challenges or inhibitors to organisational change, and developing ideas to enhance organisational capacity. The role of the Safe\&Together consultant was to guide experienced practitioners to implement principles and practices of the model in their respective organisational contexts.

Community of Practice participants ( $n=67)$ committed to:

- $\quad$ completing S\&T pre-training requirements and attending a two-day, face-to-face, S\&T-run training program;

- $\quad$ participating in monthly workshops sharing practice examples;

This article is protected by copyright. All rights reserved. 
- $\quad$ receiving coaching from S\&T consultants via video-conference;

- $\quad$ providing senior leadership, as change-agents to their teams and organisations, bringing the new knowledge and resources to secondary participants who were 'influencees' ( $n=212$ ) with identified colleagues they elected to 'influence';

- reflecting on learning, organizational change and research participation in a final focus group;

- $\quad$ completing Time 1 and Time 2 online Questionnaires; and

- $\quad$ participating in a national workshop to develop practitioner principles and guidance.

The work undertaken contributed to the co-design of practice principles by participants in the CoP through harnessing existing, albeit undocumented, good practice from the different stakeholders' perspectives with support from the S\&T Institute. The contribution of participants in the final national workshop provided the foundations for the practitioner guidance, exemplar case studies and final report. (Anonymous, 2018b \& c).

\section{Data collection and analysis}

Three data sources are drawn upon to answer the research question: a Time 1 (T1) and Time 2 (T2) online questionnaire completed by CoP participants and secondary participants; focus group data obtained after the final CoP workshop; and ethnographic notes of CoP workshops recorded by researchers.

The online questionnaire asked about respondents' perspectives of their current practice across several domains including assessment and documentation of the perpetrator's pattern of abuse and the survivor's strengths. It also included two open-ended questions: What do you think your agency is doing best in terms of working with fathers who use DV? and Where does your agency require the greatest area of improvement in working with fathers who use DV? Two hundred and seventy-six participants (including secondary participants) were sent questionnaires. Responses 
were provided by 122 respondents who responded at T1 prior to participating in Safe\&Together training and subsequent participation in the CoPs. The same respondents were asked to fill in a T2 questionnaire within six weeks of the final CoP workshop. One hundred and twelve participants responded to the two questions. A return rate of $84 \%$ was achieved from a total of 234 responses.

During each CoP session, a researcher took detailed notes about the practice examples discussed and participants' perspectives about their experience in the CoPs and as change-agents. The research team adapted Stake's multi-site research template to ensure that there was consistency across the four different states in the ethnographic note taking data (2006). Stake's work is designed specifically for research where there are multiple sites and focuses on case study and qualitative data collection including the provision of template tools. The data provided the theoretical and practical basis for synthesising the notes from the four sites (2006).

Participants attended a focus group after the final CoP where they discussed their perceptions of changes within their own practice and organisations. In some states with high numbers, two focus groups were held ( $N=6$ focus groups with 60 participants). Focus groups were audio-taped. The qualitative survey data from the two questions in the questionnaires was recorded in a table specifying each individual's response at T1 and T2. Themes were then derived from this table. Focus group data was transcribed and entered into NVivo alongside the notes bringing together all elements of the qualitative research for analysis. Thematic analysis was used to guide the data analysis using primary and then secondary coding (Thomas \& Harden, 2008). While the senior researcher took responsibility for the initial coding, constant checking between researchers led to the synthesis of the data into over-arching themes.

\section{Findings}

The findings are presented across four themes that were common across the four research sites and strongest in relation to practitioners' perspectives about the support they required from their organisations to work safely and effectively with fathers who use violence. The findings are 
aggregated across sites, presented by research method, and according to participants' service type (CP for child protection; FS for family services; DV for domestic violence services; O for 'other' services). Abbreviations for research method are referred to as follows: FG (focus groups); CoP (ethnographic notes from the Community of Practice); \#Number (questionnaire number). Ethics agreement required aggregated findings to ensure anonymity; thus, DV-S1-CoP indicates a point made by a DV practitioner in Site 1's Community of Practice session. Sub-themes have been italicised in the text and quotes have been used to exemplify themes.

\section{Senior management support}

Senior management support from within organisations was identified as critical in providing the leadership and policy settings to create the context for practitioners to change their practice and influence colleagues. The issues of worker safety and training to work with fathers who use violence are entwined with senior management support but were such strong themes they are discussed separately.

The importance of the role of senior managers in creating the levers for practice change should not be under-estimated. It was particularly noticeable in the engagement of the Program Advisory Group participants as experienced by many workers on the ground:

We have a very engaged CEO who met with participants to be briefed and is developing an organisation wide model incorporating S\&T. Management support was very noteworthy. (FS-S3-FG)

Alternatively, the lack of support was also noted:

Our Management does not seem to understand the specific risks of DV and what this means for the victim and community at large. They are the decision makers, and this is where we fall down - action is unable to be taken as the decision makers are not informed. (Other-S2$\mathrm{T} 1 \# 46)$

This article is protected by copyright. All rights reserved. 
Shifting the focus to fathers who use violence and identifying the protective strategies of mothers and the support they require refocuses DV intervention and requires considerable organisational change.

The organisation is in the process of developing training for all staff in working with fathers who use violence as a core competency within the organisation. The perpetrator pattern approach is being utilised within referral documentation and conversations across the organisation (and externally). (DV-S3-T2\#95)

Elsewhere, a worker reported:

Our Agency is sourcing a...DV program for men... This is an area which we have not been involved with in our past 25 years of operating. (DV-S1-T2\#73)

Poor leadership and lack of practice change was noted:

Management requires a greater understanding of DV - at times there is a push to include fathers without doing appropriate risk assessment. A manager attended a meeting where he recommended the father become a mentor to young children, despite previous [protection orders]. When challenged he commented "well there's family violence and then there's family violence". (FS-S3-T1\#52)

We often still hold the survivor who is most often the primary carer responsible for the care and protection of the child...[We] lose sight of who is actually causing the children to be unsafe and that this is a failure to protect. (CP-S4-T2\#69)

States with well-developed infrastructure for working with fathers who use DV demonstrated the value of senior management in advocating for, and establishing these resources.

My agency has two DV practitioners in place as consultants to case managers in order to highlight DV concerns and intervention needs, assisting case managers with risk assessment 
and safety planning and working with dads. This happens in the form of case consultations regarding families in the service where DV has been identified as high risk. (FS-S3-T1\#45)

A specific barrier for workers was heavy workloads. The role of management support was critical here. Many participants reported that their agencies and supervisors were supportive and receptive to all aspects of the project, with managers covering case-loads so they could attend workshops. Others had contrasting experiences:

Workload issues play a large part in participants having the opportunities to implement the S\&T Model into their practice and to be able to influence others. In some cases, this meant that they were unable to even attend the Invisible Practices workshops. (CP-S4-CoP)

The specifics of workload demand were mentioned by many:

In terms of $\mathrm{CP}, \mathrm{I}$ understand that the core business is child safety and due to tight [key performance indicators], timelines and volume of workload, practitioners tend to concentrate on the mother and her ability to 'protect' the child. CP practitioners need more practice understanding the value of reaching fathers and spending time examining their behaviour over time. (CP-S3-T1\#174)

Practitioners named a wide range of ways in which senior managers and organisational infrastructure influenced the opportunity to focus greater attention on the father using violence and in partnering with the child's mother.

\section{Support for organisational collaboration}

Practitioners were effusive in describing improved inter-agency practice through the implementation of the Safe\&Together Model. In relation to the collaboration between statutory child protection and specialist DV services, a DV practitioner described developing trust:

When I came into the sector there was a lot of distrust ... If we would share with... [CP] we often got knee-jerk reactions or reactions that totally exposed the victim, and put her at high 
risk, so as a sector we closed the door and we no longer talked to...[CP] because it was not safe to do so. But since [S\&T] Model has come in ... the trust has been built. (DV-S2-FG)

A child protection practitioner remarked on their improved partnering with the adult victim/survivor through strengthened collaboration with a specialist DV service:

We are on the same page - she knows we are in her [survivor's] corner. We advocated together to get the [protection order] in partnership with the women's service. We are bringing it to the table - she can see that I'm not trying to sabotage her. (CP-S1-FG)

Participants pointed to the trust that underpins information-sharing to support collaborative practice. All states have information-sharing legislation to guide practice, but it was noted that community sector organisations and $\mathrm{CP}$ workers were now more open to sharing information than previously:

I have been in meetings where they [CP] have always left or they sit reserved or don't liaise with other services. They don't say anything. Silence is power. (DV-S1-FG)

While participants in another state recognised that information-sharing had progressed at formal case management meetings, another state had developed one Information Sharing document to which all professionals working with a family contributed. However, the need to provide further information on referrals was a noted area for improvement raised in practice examples across CoPs.

The perpetrator was continually sabotaging the survivor's efforts to abstain from substance use, yet the referral for the survivor to attend substance use counselling did not have any information pertaining to the perpetrator's behaviours. (CP-S4-CoP)

CoP practitioners discussed the potential to break down collusion with the perpetrator when organisations were working well together. By sharing information and supporting each other, a practitioner believed that perpetrators were left with "no way to manipulate the situation." (DV-S3CoP) 
In summary, when statutory services are involved, collaborations must have the formal support of organisations' senior management. However, collaboration went beyond formal procedures to emphasise the importance of trust between workers and their respective organisations particularly when working with fathers who use violence.

\section{The centrality of combined training, coaching and supervision}

The need for further training to develop the skills to work with fathers who use violence and to partner with women survivors was a ubiquitous theme. A word search in response to the questionnaires' open-ended questions, asking workers what their organisation did well and where their organisation could improve. Eighty-seven references to training were made. More specifically, numerous references were made to the need for all workers to be trained to work with fathers who use violence.

The organisation is in the process of developing training for all staff in working with fathers who use violence as a core competency within the organisation. (DV-S3-T1\#95)

Where secondary participants mainly identified the need for 'training' as a key driver for improving practice, CoP participants highlighted combined Safe\&Together training, coaching and resources alongside peer-to-peer support as key drivers for learning. The Safe\&Together resources stress the ways to engage men as fathers to reflect on their parenting practices. Participants of one focus group expressed the view that the regularity of the workshops was integral to practice development and organisational learning through the sharing of different albeit allied perspectives. They reported that the CoP process with ongoing S\&T training was:

...more effective than individual training as it addressed discrepancies in training and experience levels; for example [it]...allowed workers to develop consistency in recording case-notes and focussing on perpetrator behaviours. (whole-S2-FG)

This article is protected by copyright. All rights reserved. 
Finally, results from the $\mathrm{T} 1$ and $\mathrm{T} 2$ questionnaires demonstrated that many workers overestimated their initial starting competency:

Training showed me that we weren't necessarily aware of ourselves and we rated ourselves more highly than we might have done... we may have thought we were doing a better job than we were. (FS-S3-FG)

\section{Risk assessment and management to support worker safety}

Practitioners stressed that increased attention to fathers who use violence requires a parallel increase in attending to worker safety. The Safe\&Together consultant crystallised discussion by articulating that practitioners' fear for their own safety and that of survivors shows up in "subtle and not so subtle ways" (S\&T-S2-CoP). Their strategies often mirror adult and child survivors' strategies for managing safety, such as not involving police and avoiding topics that may escalate harmful behaviours:

When we pay attention to the worker safety issue, if we watch it really closely, we actually can learn a lot about the experience of adult and child survivors managing safety. (S\&T-S2CoP)

Differences and tensions emerged in CoP discussions between the statutory and community sector participants owing to their legislative responsibilities and to where and how they engage with perpetrators. For example, one statutory service's practitioner observed that worker safety is easier for them to manage because their work is office-based; whilst others observed they are required to enter dangerous situations at times without the freedom to withdraw:

It's a different mindset...we [CP] have to do home visits while DV services [say] 'we won't go into the home'. (CP-S3-CoP)

Tensions between community organisations were illustrated by another participant involved in men's behaviour change programs who observed that 
We conduct groups and they're in relatively safe, controlled environments. The men's sector needs to do work to understand the other sectors, such as child protection and family service workers. (DV-S3-CoP)

Organisations generally had policies on worker safety with most CoP participants identifying strategies including duress alarms, joint visits, and working alongside police on high risk matters. The translation of safety into practice was critical with examples of poor credence given to the requirement for joint visits as the default protocol in certain high-risk situations. For example, in the hundreds of clients visited by one practitioner's organisation in the previous year there had been no joint visits. She indicated that "a lack of adequate funding... [meant] there were no worker safety considerations" (FS-S1-CoP). As the S\&T consultant advised in several CoPs, keeping the focus on perpetrators requires a priority on worker safety: "...that working in multi-disciplinary teams is a great way to manage safety."

Practitioners acknowledged an important distinction between physical and emotional safety. Child protection participants across CoPs were concerned that attention to emotional safety is overlooked in comparison to physical safety. Participants across sites mentioned "vicarious trauma" being used, in the words of one practitioner, "...to shame workers" (CP-S1-CoP). Another said:

Not many organisations could say they manage the emotional side of their employees very well. There's a stigma to it as well...they think about the physical side, but the emotional side is often forgotten. (CP-S2-FG)

Participants discussed the vital need for quality supervision that enabled workers to adequately debrief and critically reflect on the nature of the work when engaging with men who use violence. In a discussion about the comparative youth and gender of the child protection workforce, one group touched on gaining knowledge from post-visit supervision insights: 
Sometimes it's not until after visits that you realise the risks you're facing. It's important for [these] system supports to be in place... (DFV-S3-FG)

In addressing organisational culture and attitudes to worker safety, participants in several states acknowledged the 'normalisation' of clients' abusive text and phone communications and the accompanying 'desensitisation' that occurred. Quality supervision was considered a prerequisite to address such issues, not only to pivot to the perpetrator and partner with women but as a way of ensuring that the safety, wellbeing and upskilling of practitioners, particularly newly qualified, were attended to.

Participants argued that it was not possible to undertake work that focused on fathers who used violence without a range of strategies that attended to the organisational culture in relation to the physical and psychological safety of workers.

\section{Discussion}

The themes that child protection, and DV workers raised about the organisational support they required to institute practice changes for working with fathers who use violence are not new: senior management leadership; acknowledgement of the need to refocus the work in the DV area; prioritising worker training, coaching and peer support; and greater attention to worker safety. These are broad categories. However, it was the detail of the organisational change context and the responses bought to the CoP which contributed to the co-design of principles for practice when working with fathers who use violence (Anonymous, 2018). This practice-led knowledge building highlighted the interplay between practice and context which privileges the worker experience. It looks to the ways in which practitioners build new knowledge based on their practice within the context in which they work (Wagenaar \& Cook, 2011; Howard \& Somerville, 2014).

Practitioners acknowledged that child protection and family services practice where children were impacted by DV needed to change substantially. This is an early step in a co-design process and support for organisational change. Poor practices included: ignoring the perpetrator of violence 
(usually the biological or social father); focusing all practice on the child's mother; assessing the mother's deficits; her ability to protect her children; and demanding separation as the only route to safety. These practices were identified as long-standing but destructive (Anonymous 2018).

The authorising environment to facilitate a context for change to address these often harmful practices requires leadership from senior managers in both the statutory and community sectors (Gilley et al, 2009). Whilst some senior managers were prepared to drive the organisational change process (Gill, 2003), others were merely supportive of innovative work without leading change. The latter 'hands off' approach made it difficult for practitioners to embed change and highlighted the need for proactive senior management leadership particularly in an area where practices have become entrenched. The focus on mothers and their 'failure to protect' their children, or problems with mental health or alcohol and other drug problems (often connected to the abuse they are experiencing) (Lapierre, 2010), have constantly shifted the attention from fathers and their violence, abuse and harm to children in the context of domestic violence.

Practitioners' strongest message was that they recognised that working with fathers who use violence is skilled work for which they feel ill-prepared. This is a sentiment echoed elsewhere where it is noted that working with men who use violence is not a core learning skill in the training qualifications for social workers and human service professionals (Scourfield et al, 2015). Many new skills are involved, for example: working with men to shift their focus from blaming their partners for all family problems to the man's own parenting behaviours; the ability to be assertive, but respectful and non-collusive; moving from a focus of belief and validation, to suspending belief while information emerges from others; and undertaking sensitive and comprehensive DV risk assessment and risk management were all issues covered in the Communities of Practice. It was also recognised that it was not possible to work effectively and safely with men who use violence as fathers without also building a partnership with the man's partner or ex-partner. 
The experience of a comprehensive approach to learning and development through the Invisible Practices project highlighted the inadequacies of a reified model of individual training. Cook and Wagenaar are particularly critical of the individualised approach to training:

Work stops, a period of training ensues (frequently offsite...), after which employees return to the workplace to put new knowledge into practice (not always with sterling success). Billions are spent annually on training following this model (2012, p.25).

For those working in the DV area there has been a consistent message that training a workforce to intervene differently in DV requires commitment of the whole organisation (Featherstone \& Peckover, 2007; Stover \& Morgos, 2013). Supervision and coaching are needed to support practice change (Anonymous, 2018d) particularly in the undeveloped area of working with fathers who use violence. In the project, intensive online and face-to-face training was complemented with coaching provided in the CoPs over a six-month period. Each participant was also expected to 'influence' their colleagues in their organisation and promote practice improvements. Practitioners spoke enthusiastically about this embedded approach to learning and the ripple effect this could have through their organisation. This approach supports the reciprocal role that practitioners can play in influencing the organisational context (Cook \& Wagenaar, 2012) and the co-design of interventions (Evans \& Terrey, 2016).

The original design of the research project recognised the reciprocity between practice and context or 'top down' and 'bottom up' interventions to facilitate change. Indeed, this recognition underpinned the establishment of a Program Advisory Group of senior managers in each state. While the researchers had recognised a role for senior managers, it was participants in the CoPs who constantly highlighted the primacy of senior management support. It was recognised that individual practitioners alone were not going to change practice. Rather, a whole of organisation approach was required to facilitate this change process (Osbourne, 2014). Senior management involvement to 
facilitate change processes thus emerged as a central co-design principle for changing practices with fathers who use violence.

Most importantly, senior management needed to recognise that a single organisation does not have an exclusive hold on effective responses to children living with domestic violence. The work must be collaborative and inclusive of a range of justice and human service organisations in responding to men, women and children (Allen, 2006). The divisions between child protection workers, family and specialist DV workers have been longstanding (Lapierre, 2010) and provided a focus for the co-design work of this project. Finding a language and direction for change (the first stage of a co-design process) (Evans \& Terrey, 2016) was assisted through the Safe\&Together training where the framework gave practitioners from across sectors a shared vision (Anonymous 2018a) and provided a major contribution to facilitating multi-agency working in the DV area more generally.

Across all organisations, attention to worker safety when responding to DV is accepted as an important aspect of the work. No statutory or specialist DV service would be without its Occupational Health and Safety policy. However, as workers in this project and elsewhere have pointed out, organisational culture in relation to worker safety is critical (Littlechild \& Burke, 2006). Physical safety issues were raised specifically in relation to risk assessment of perpetrators, joint visits, and the vulnerabilities of new and female workers, all familiar themes in this area (Jenney et al, 2014). However, the area of emotional safety and the vulnerability to vicarious trauma that practitioners identified point to the need for regular clinical supervision and debriefing. The problems associated with a 'macho' culture which characterised fear and emotional vulnerability as 'weakness' or a sign of worker unsuitability for the job were noted as particularly destructive aspects of some organisational cultures.

\section{Conclusion}

This article is protected by copyright. All rights reserved. 
Shifting the focus of practice to address the harm that fathers who use violence do to their children requires an organisational context which supports and backs this change in practice. Workers from across child protection, family services and specialist DV collaborated at front-line, team leader and senior management level and demonstrated a model for implementing a co-designed model for change. Danger and risk increases for workers who intervene with fathers who use violence. It is work for which many workers feel ill-prepared.

Practitioners' understanding of the constraints and facilitators of practice change within organisations validates the significant role that practice plays in knowledge development. This conceptualisation "turns...on its head" the notion of workers applying already established knowledge in practice (Cook \& Wagenaar, 2011, p. 193), and instead recognises that they can be primary contributors to knowledge development. Their messages are succinct and clear and challenge senior managers to take up their role in facilitating the change agenda.

This article is protected by copyright. All rights reserved. 


\section{References}

Anonymous, 2015

Anonymous, $2017 a$

Anonymous 2017b

Anonymous,2018a

Anonymous, $2018 \mathrm{~b}$

Anonymous, 2018c

Anonymous, 2019,

Allen, N 2006, 'An examination of the effectiveness of domestic violence coordinating councils', Violence Against Women, vol. 12, pp. 46-67.

Blackmore, C 2010, Social Learning Systems and Communities of Practice, Springer, London.

Buchanan, F 2018, Mothering babies in domestic violence: Beyond attachment theory, Routledge, London.

Cook, N \& Wagenaar, H 2012, 'Navigating the Eternally Unfolding Present: Toward an Epistemology of Practice', The American Review of Public Administration, vol. 42, pp. 3-38.

Cox, P 2016, 'Violence against women: Additional analysis of the Australian Bureau of Statistics' Personal Safety Study, ANROWS, Sydney.

Coy, M, Thiara, R, Kelly, L, \& Phillips, R 2011 'Into the foreground: an evaluation of the Jacana $\begin{array}{llll}\text { Parenting } & \text { Program', } & \text { viewed } & \text { March }\end{array}$ http://www.niaendingviolence.org.uk/perch/resources/into-the-foreground-jacana-evaluation-

report.pdf.

This article is protected by copyright. All rights reserved. 
Evans, M \& Terrey, N 2016, 'Co-design with citizens and stakeholders', In G. Stoker \& M. Evans (eds.), Evidence-based policymaking in the social sciences, Policy Press, Bristol.

Featherstone, B \& Peckover, S 2007, 'Letting them get away with it: Fathers, domestic violence and child welfare, Critical Social Policy, vol. 27, no. 2, pp. 181-202.

Gill, R 2003, 'Change management-or change leadership?' Journal of Change Management, vol. 3, pp. 307-321.

Gilley, A, McMillan, H \& Gilley, J 2009, 'Organizational Change and Characteristics of Leadership Effectiveness', Journal of Leadership and Organisational Studies, vol. 16, pp. 38-47.

Gustavsson, S \& Andersson, T 2017, ‘Patient involvement 2.0: Experience-based co-design supported by action research'. Action Research, pp. 1-23.

Healy, K 2017, The Skilled Communicator in Social Work: The Art and Science of Communication in Practice, Palgrave, Basingstoke.

Holt, S, Buckley, H, \& Whelan, S 2008, 'The impact of exposure to domestic violence on children and young people: A review of the literature', Child Abuse and Neglect, vol. 32, pp. 797-810.

Holt, S 2015, 'Post-separation fathering and domestic abuse: challenges and contradictions', Child Abuse Review, vol. 24, pp. $210-222$.

Howard, Z \& Somerville, M 2014, 'A comparative study of two design charrettes: implications for codesign and participatory action research', International Journal of Co-Creation in Design and the Arts, vol. 10, pp. 46-62.

Ison, R 2017, Systems practice: How to act. $2^{\text {nd }}$ edn, Springer, London.

Ison, R 2008, 'Systems thinking and practice for action research', in P Reason \& H Bradbury (eds.), The Sage Handbook of Action Research: Participative inquiry and practice 2nd edn, Sage Publications, London, pp. 139-158.

This article is protected by copyright. All rights reserved. 
Jenney, A, Mishna, F, Alaggia, R, \& Scott, K 2014, 'Doing the right thing? (Re) Considering risk assessment and safety planning in child protection work with domestic violence cases', Children and Youth Services Review, vol. 47, pp. 92-101.

Lapierre, S 2010, 'Are abused women "neglectful" mothers? A critical reflection based on women's experiences', in B. Featherstone, CA Hooper, J Scourfield, \& J Taylor (eds.), Gender and Child Welfare in Society, Wiley-Blackwell, Chichester and Hoboken, NJ.

Littlechild, B \& Bourke, C 2006, Men's use of violence and intimidation against family members and child protection workers. In N. Stanley (eds.), Domestic violence and Child Protection: Directions for Good Practice. Jessica Kingsley, London.

Mandel, D 2014, 'Beyond domestic violence perpetrator accountability in child welfare systems, No to Violence Journal, vol. 1, Spring, pp. 50-85.

Mandel, D 2009, 'Batterers in the lives of their children', in E. Stark \& ES. Buzawa (eds.), Violence Against Women in Families and Relationships, Praeger/ABC-CLIO, Santa Barbara, CA.

May, C 2013, 'Agency and implementation: Understanding the embedding of healthcare innovations in practice' Social Science \& Medicine, vol. 78, pp. 26-33.

McConnell, N \& Taylor, J 2016, 'Evaluating programs for violent fathers and ethical review', Child Abuse Review, vol. 25, pp. 183-191.

McTavish, J. R, MacGregor, J, Wathen, N, \& MacMillan, L 2016, 'Children's exposure to intimate partner violence: an overview', International Review of Psychiatry, vol. 28, pp. 504-518.

Nygren, K, Walsh, J, Ellingsen, I, \& Christie, A 2019, 'What about the fathers? The presence and absence of the father in social work practice in England, Ireland, Norway, and Sweden - A comparative study'. Child and Family Social Work, vol. 24, pp. 148 - 155.

This article is protected by copyright. All rights reserved. 
Osbourne, M 2014, 'Working with fathers to safeguard children: Failure to work with fathers around the child occurs regularly', Child Abuse \& Neglect, vol. 38, pp. 993-1001.

Riviara, F, Anderson, M, Fishman, P, Bonomi, A, Reid, R, Carrell, D \& Thompson, R 2007, 'Intimate partner violence and health care costs and utilization for children living in the home', Pediatrics, vol. 120 , pp. $1270-1277$.

Safe\&Together Institute 2018, Welcome to the Safe \& Together Institute, viewed May 1, 2018, https://safeandtogetherinstitute.com/ Scourfield, J, Smail, P, \& Butler, D 2015, 'A Systemic approach to improving the engagement of fathers in child safeguarding', Child Abuse Review, vol. 24, pp. 129139.

Stake, R 2006, Multiple Case Study Analysis, Guilford Press, New York.

Stover, C \& Morgos, D 2013, 'Fatherhood and intimate partner violence: Bringing the parenting role into intervention strategies', Professional Psychology: Research and Practice, vol. 44, pp. 247.

Thomas, J. \& Harden, A. 2008, 'Methods for the thematic synthesis of qualitative research in systematic reviews, BMC Medical Research Methodology, 8:45. doi:10.1186/1471-2288-8-45

Thompson-Walsh, C, Scott, K, Dyson, A \& Lishank, V 2018, 'Are we in this together? Post-separation co-parenting of fathers with and without a history of domestic violence', Child Abuse Review, vol. 27, no. 2, pp. 137-149.

Wagenaar, H \& Cook, N 2011, 'The push and pull of the world: how experience animates practice', Evidence \& Policy, vol. 7, pp. 193-212.

Witt, L \& Diaz, C 2019, 'Social workers' attitudes' towards female victims of domestic violence: A study in one English local authority', Child and Family Social Work, vol. 24, pp. 209-217.

This article is protected by copyright. All rights reserved. 


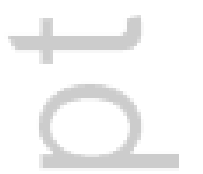

4 


\section{University Library}

\section{- M M I E E R VA A gateway to Melbourne's research publications}

Minerva Access is the Institutional Repository of The University of Melbourne

Author/s:

Humphreys, C;Healey, L;Heward-Belle, S

Title:

Fathers who use domestic violence: Organisational capacity building and practice development

Date:

2019-11-13

Citation:

Humphreys, C., Healey, L. \& Heward-Belle, S. (2019). Fathers who use domestic violence: Organisational capacity building and practice development. CHILD \& FAMILY SOCIAL WORK, 25 (S1), pp.18-27. https://doi.org/10.1111/cfs.12708.

Persistent Link:

http://hdl.handle.net/11343/286933 